\title{
BUILD-IT: A brick-based, integral solution supporting multidisciplinary design tasks
}

Morten Fjeld ${ }^{1}$, Kristina Lauche ${ }^{2}$, Stefan Dierssen, Martin Bichsel ${ }^{3}$ \& Matthias Rauterberg ${ }^{4}$

${ }^{1}$ Institute for Hygiene and Applied Physiology (IHA), ETH Clausiusstr. 25, CH-8092 Zurich, SWITZERLAND phone: +41-1-6323983, fax: +41-1-6321173 e-mail: fjeld@iha.bepr.ethz.ch

${ }^{2}$ Institute for Work and Organisational Psychology (IfAP), ETH Nelkenstr. 11, CH-8092 Zurich, SWITZERLAND phone: +41-1-6326142, fax: +41-1-6321186 e-mail: lauche@ifap.bepr.ethz.ch

${ }^{3}$ Institute for Design and Construction Methods (IKB), ETH Tannenstr. 3, CH-8092 Zurich, SWITZERLAND phone: +41-1-6322429, fax: +41-1-6321181 e-mail: \{dierssen, mbichsel\}@ikb.mavt.ethz.ch

${ }^{4}$ Center for Research on User-System Interaction (IPO), TUE NL-5612 AZ Eindhoven, NETHERLANDS phone: +31-40-2475242, fax: +31-40-31930 e-mail: rauterberg@ipo.tue.nl

\section{Abstract}

BUILD-IT is an up-and-running system putting at work highly intuitive, videobased interaction technology to support complex planning and configuration tasks. It makes state-of-the-art computing and visualisation available to all kinds of users, without requiring any special computer literacy. Based on real, tangible 
bricks as interaction handler, BUILD-IT represents a novelty to HumanComputer Interaction. With this tool, object manipulation and image display take place within the very same working area. Hence, new dimensions of prehension and direct response have been added to Human-Computer Interaction. Technology has a back-stage position, whereas creativity and human communication within multi-disciplinary expert teams is encouraged.

\section{Keywords}

Brick-based interaction, tangible objects, intuitive planning and design

\section{INTRODUCTION}

The concept of BUILD-IT is based on highly intuitive, video-based interaction technology, supporting complex planning and configuration tasks. It allows a group of people to sit around a table and handle projected objects with real tangible bricks as the interaction handler. Computer Aided Design (CAD)-based objects are manipulated and displayed within the very same working area. Hence, a new dimension of prehension and direct response has been added to HumanComputer Interaction (HCI). Dynamically coupled with an image displayed on the table, a perspective view of the planning situation is projected on a screen. This system makes state-of-the-art computing and visualisation available to all kinds of users, without requiring any special computer literacy. Instead of dominating cognitive and social planning processes, the system actually supports creativity and human communication.

For most planning tasks in systems engineering and architecture, drawings and 2D models have been replaced by CAD applications. This change has brought about a range of supportive tools for drawing and information processing. However, it also implies less immediate contact among CAD users, planning experts and sales people.

We began our work by performing a task analysis with potential user groups for our system. We observed that they spent a great deal of time in discussions with their clients and noticed that off-line CAD support is hardly available during sales trips. This lack of support sometimes caused misunderstandings with the designers 'at home', trying to communicate their solutions to the travelling sales people. Also, some of the customers were not familiar with 2D layout techniques; they were unable to imagine what an object would look like in 3D. Therefore, an easy-to-handle, 3D-planning tool proved to be of high interest to planning experts and to sales people. A distributed, networked system would additionally allow for interaction among users located at different sites. 
Actually, modern management concepts like Simultaneous Engineering are based on dynamic interaction among co-operating experts. In this context, a tool should encourage team co-operation rather than each person working in front of a separate screen. Such needs can hardly be met by existing technologies like video conferencing. An adequate solution has to offer more intuitive, natural interaction.

All these considerations were taken into account in the design process of BUILDIT. A system bringing support to early offering and design processes was the result. This tool is not intended as an alternative, but rather as a complementary aid for CAD systems. It allows for ready-made applications in various field, such as machine configuration, city and urban planning, architecture and interior design.

Tangible bricks represent a new way of interaction. Among others, this type of interaction was described by Ishii and Ullmer (1997), Underkoffler and Ishii (1998) and Fjeld, Bichsel and Rauterberg (1998). Rauterberg, Mauch and Stebler (1996) showed that a brick based interface is significantly easier and more intuitive to use than mouse and screen based interfaces.

Compared with physical, model-based layout systems, BUILD-IT additionally offers handling of CAD-objects and data management. It features cheaper, quicker and more exact object representation. The potential of computer mediated work is made readily available through automatic calculation of prices and time-to-delivery. Two-way communication with external CAD systems is assured, whereas animation and simulation offer design support at an expert level.

Offering higher efficiency in human communication, the system enables designers to accomplish their job with less travelling in less time. Distributed, networked systems offer simultaneous interaction for users located at different sites. Networked systems also encourage spontaneous distributed interaction, going far beyond the traditional, computer-based concept of teamwork.

In this paper, we will first give a system description, followed by an in-depth presentation of how geometric and meta-data is used by the system. Finally, we describe some user experiences. 


\section{SYSTEM DESCRIPTION}

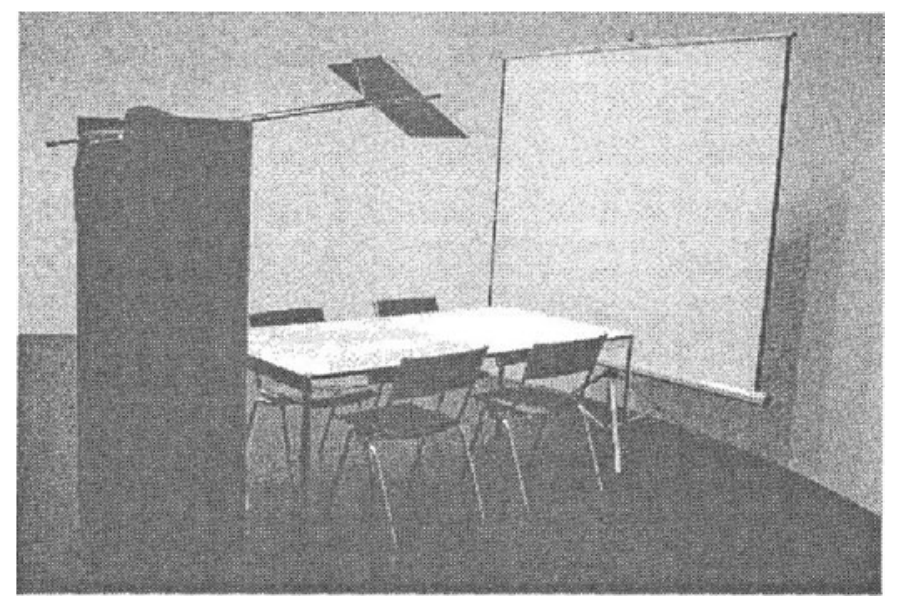

Figure 1: BUILD-IT.

In a first step, we have designed a partial Natural User Interface (NUI) instantiation (Figure 1), as described by Fjeld, Bichsel and Rauterberg (1998). Partial means that distributed communication between multiple systems has not yet been implemented. As the task context, we chose that of planning activities for plant design. A system, called BUILD-IT, was realised. This is an application that supports engineers in designing assembly lines and building plants.

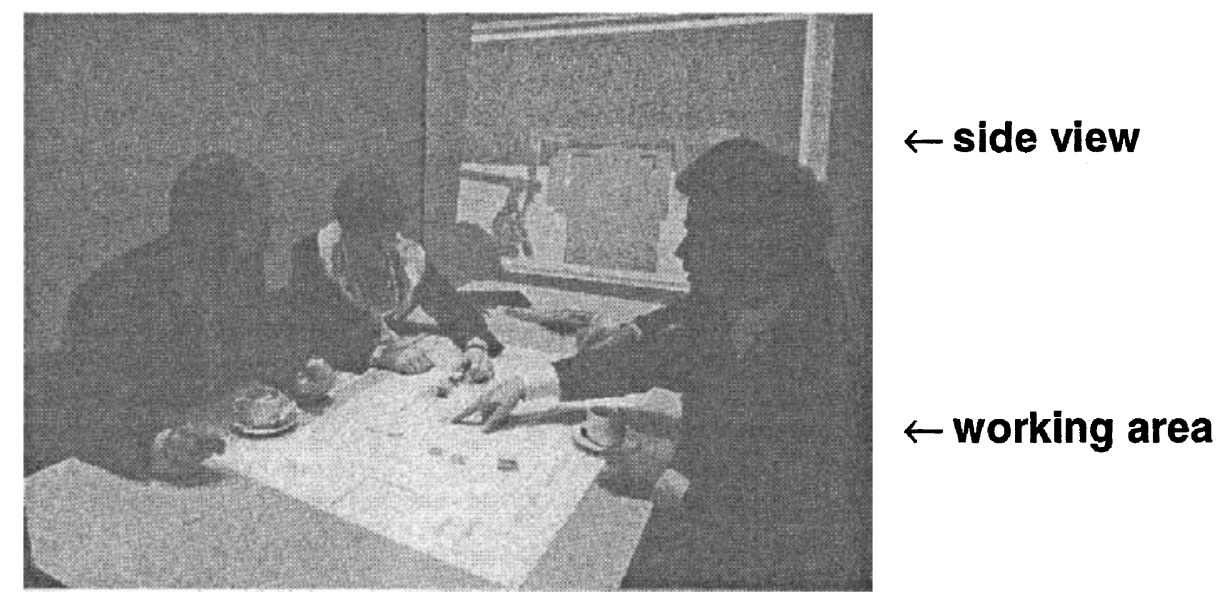

Figure 2: Typical task solving situation with BUILD-IT. Interaction and display take place in the working area, whereas a perspective is offered by the side view.

The system enables users, grouped around a table, to interact in a space of virtual and real world objects. On the screen, a side view (Figure 2) offers a perspective of the planning situation. In the working area (Figures 2 and 4) there is an above view (planning situation as seen from above), height view (a slice of the side 
view, for object height adjustment) and menu (split into a left and right part, offering new objects and functions). In the above view, height view and menu, objects can be selected, positioned, rotated and fixed (Figure 3). Functions (objects with functionality, like virtual camera, scaling, save \& print) are selected in the menu and can be used in the above view.

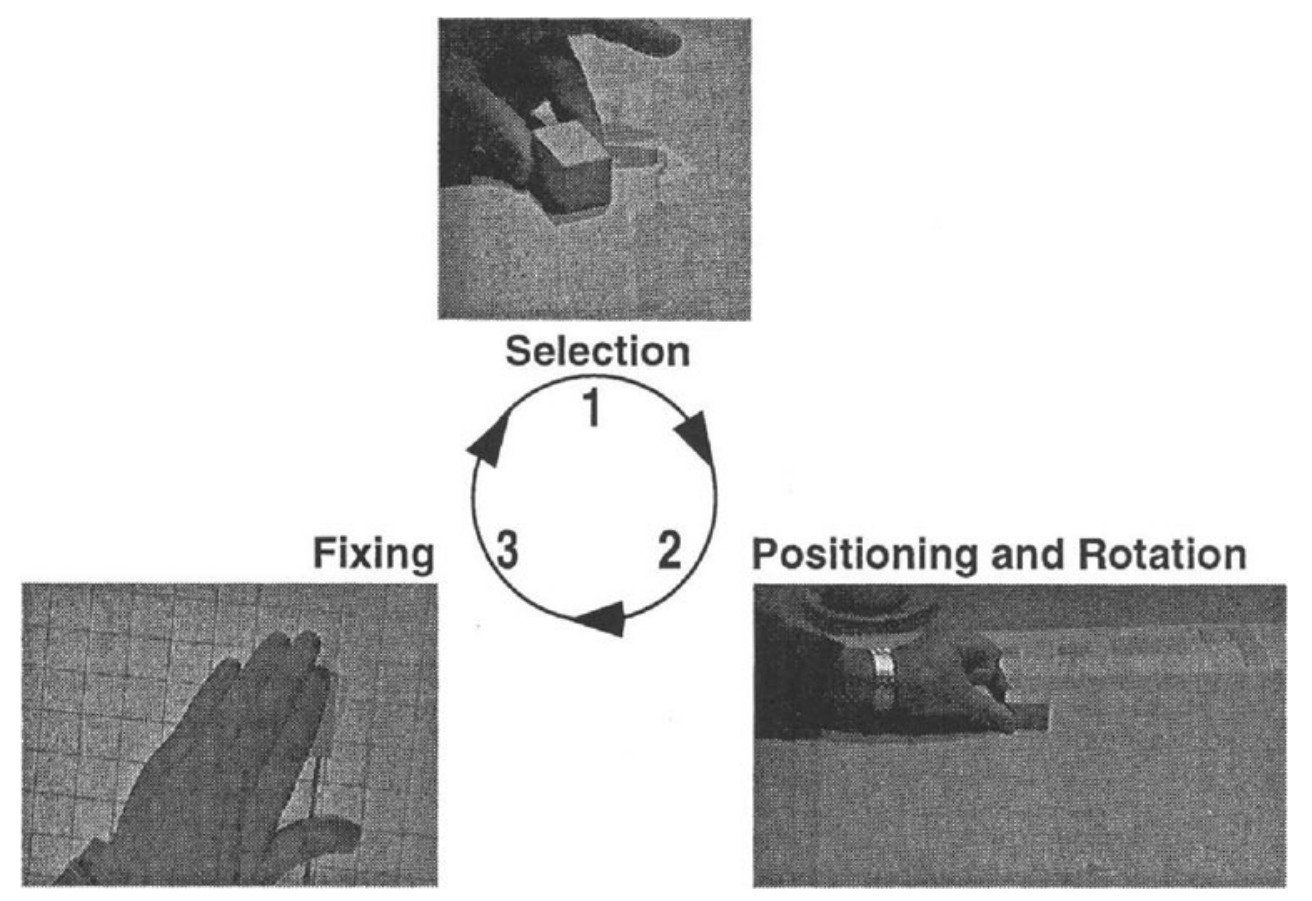

Figure 3: The basic steps for user manipulations with the interaction handler.

The basic principle of BUILD-IT is shown in Figure 3. Users select an object by putting the brick at the object positions. The object can be positioned, rotated and fixed by simple brick manipulation. Using a material brick, everyday motor patterns like grasping, moving, rotating and covering are activated. Throughout these steps, there is a strong connection between cognitive processing and observable behaviour. The system dynamically supports the user needs for goal setting, planning, action and control. Hence, complete regulation of the working cycle (Hacker, 1994) is assured. The cost of making a mistake is low, since all vital operations are reversible. So, epistemic and pragmatic action (Kirsh and Maglio, 1994) are equally encouraged. To allow two handed operation, the system supports multi-brick interaction. A second effect of multi-brick interaction is that several users can take part in a simultaneous design process. Altogether, the set of NUI guidelines formulated by Fjeld, Bichsel and Rauterberg (1998) have been met. 


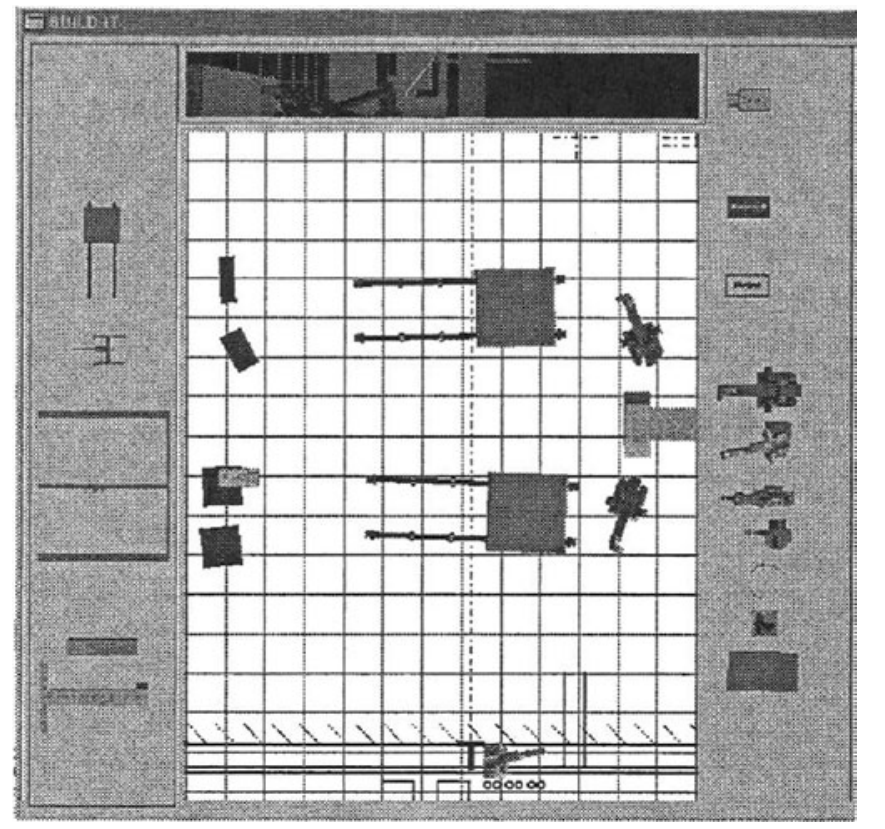

\section{$\leftarrow$ virtual camera}

$\leftarrow$ scaling

$\leftarrow$ save \& print

Figure 4: The working area with above view (situated in the centre), height view (situated on top) and menu (split into a left and right part, situated accordingly). The menu contains objects (robots, tables, conveyor belts etc.) and functions (objects with functionality; virtual camera, scaling, save \& print etc.).

The application is designed to support providers of assembly lines and plants in the early design processes. Graphical display is based on the class library MET++ (Ackermann, 1996). The system can read and display arbitrary virtual 3D objects as seen in Figure 4. These objects are sent from a CAD system to BUILD-IT using Virtual Reality Modelling Language (VRML). Geometry is not the only aspect of product data. There is a growing need to interact in other dimensions, such as cost, configurations and variants. Therefore, the system has been engineered to send and receive numerous forms of meta-data.

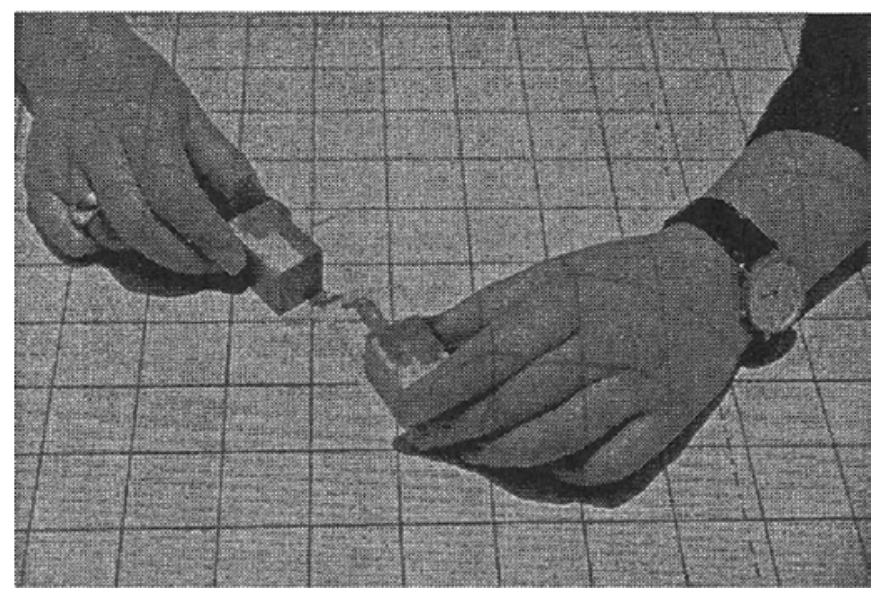

Figure 5: Multiple bricks allow for two-handed interaction . 
BUILD-IT currently features the following user (inter-) actions (Figure 2-5):

- Selection of a virtual object (e.g. a specific machine) in a virtual machine store by placing the interaction handler onto the projected image of the machine in the object menu.

- Positioning of a machine in the virtual plant by moving the interaction handler to the preferred position in the plant layout of the above view.

- Rotation of a machine by coupling machine and brick orientation.

- Fixing the machine by manually covering the surface of the interaction handler and then removing it.

- Re-selection of a machine by placing the interaction handler onto the specific machine in the above view.

- Removing the machine by moving it back into the object menu (the virtual machine store).

- Modification of object size and height by operators in the method menu applied on objects in the above view.

- Direct modification of object altitude in the height view.

- Automatic docking of two or more objects along predefined contact lines within the above view.

- Scrolling of above view, height view and menus.

- Modification of the perspective in the height and side views by a virtual camera in the above view. Numerous virtual cameras, each representing a distinct perspective, can be used at a time. The last one selected determines the current perspective.

- Saving of the working area contents by a method menu icon.

- Printing of the views, also offered by a method menu icon.

- Multi-brick and multi-person interaction. All the previous (inter-) actions can be simultaneously executed by any of the bricks at the table.

- Simulation mode, supported by a simulation software (AESOP GmbH, 1997), shows real-time manufacturing. Steel sheets can be followed as they pass through different processes, like laser welding, chemical baths and drilling.

\section{WORKING WITH VRML DATA AND META-DATA}

The BUILD-IT system understands two different 3D-CAD data formats: VRML data and meta-data. We will pay most attention to VRML data, because they describe the complete geometry and visual characteristics of an object.

Additionally, depending on the field of application, users also need auxiliary object information. First, if configuration cost of the currently handled object is of interest, product name and unit price may be required. Second, in the case of process simulation (e.g. welding of metal sheets), different objects (e.g. robot, welding- or cleaning machine) and their characteristics (e.g. machine type, 
capacity, preparation-, processing- and welding time) are needed. In both cases, object specific numbers and figures, named meta-data, are required. Such information is treated as separate data structure(s), and stored as metainformation (".mif") files.

Data exchange between a 3D-CAD system and BUILD-IT can be handled in two different ways: i) by the CAD-connection, and ii) by the Product Data Management (PDM)-connection.

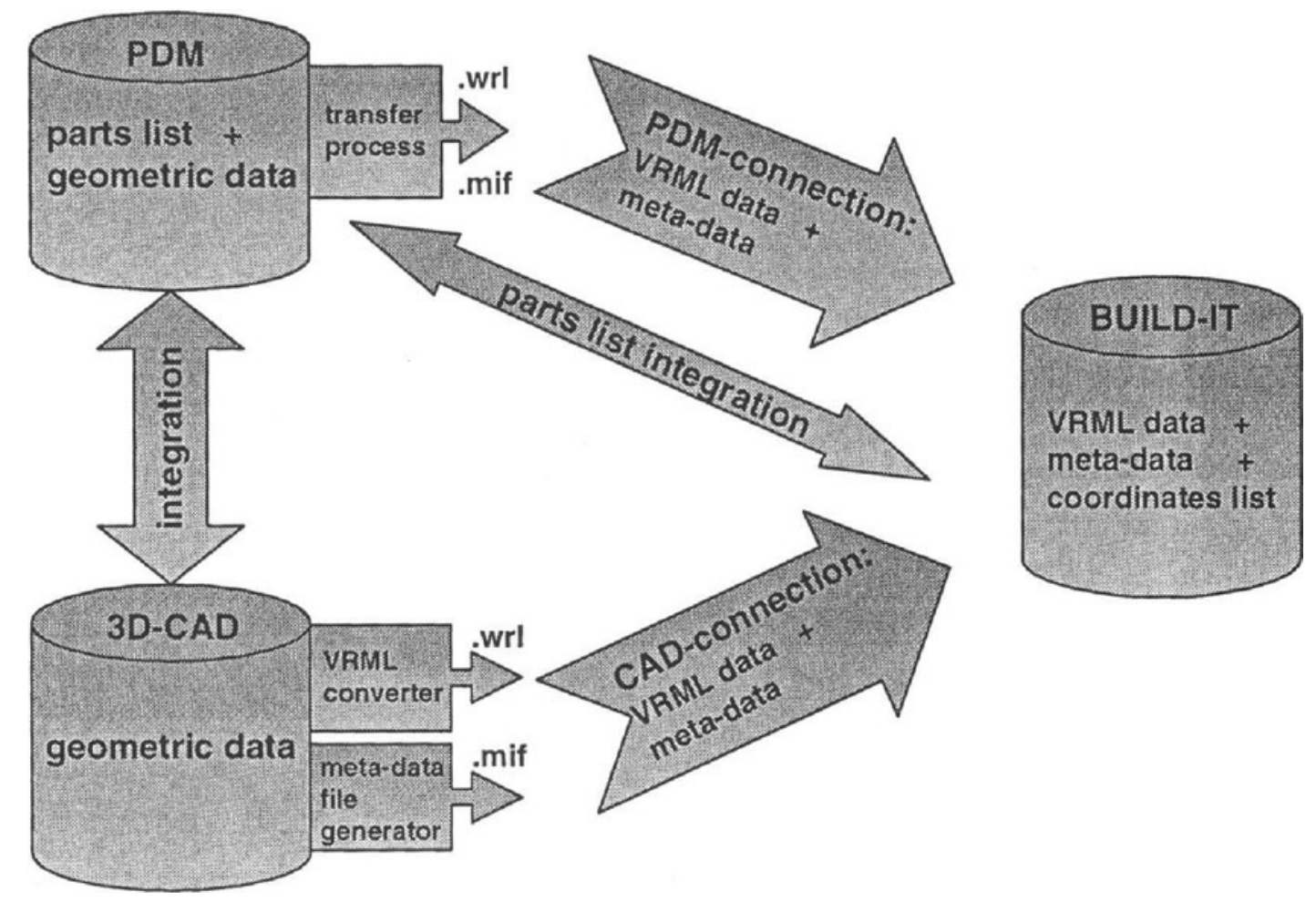

Figure 6: Data flow between the 3D-CAD, the PDM and the BUILD-IT system is based on CAD-connection, PDM-connection, integration and parts list integration.

\subsection{CAD-CONNECTION}

The most direct connection between a 3D-CAD system and BUILD-IT is the CAD-connection, as shown in Figure 6. CAD users are presented with a list of all available objects and can select the geometric data required for their specific planning session. The selected geometric data is converted to VRML format and offered by the CAD system as world (".wrl") files. Using the CAD-connection, the selected geometric data is then sent as ".wrl" files to BUILD-IT. For each 
".wrl" file, a ".mif" file is generated. A ".mif" file contains additional object information like unit price and simulation parameters.

A VRML based connection offers the important advantage of data compression, allowing for reduced information flow and less object complexity. This feature is just as vital to object handling in the Web as with the BUILD-IT system. Without data reduction, only high performance CAD systems would be able to deliver multiple 3D object within acceptable time.

However, conversion, i.e. data compression and complexity reduction, also induce serious limitations. Circles are displayed as multi-edge polyhedrons, preventing an exact geometric object interpretation. Users wishing to position one object along the tangent of a second, circular object, with millimetres precision, can no longer be supported. A further consequence of data conversion is that direct feedback from BUILD-IT to the CAD system cannot be offered. Such feedback is impossible, because exact volume and surface information gets lost through conversion, and the original parts of an object can no longer be reconstructed. For this very reason, bi-directional communication of geometric and configuration data is not possible with the direct CAD-connection.

To make the description of the CAD-connection complete, we mention that metadata, in this case ".mif" files, are also communicated via this connection. Since meta-data is exclusively being used by the BUILD-IT system, no feedback is needed, so the one-way CAD-connection is fully sufficient in the context of meta-data.

\subsection{PDM-CONNECTION AND INTEGRATION}

A more elaborate way to connect BUILD-IT with CAD systems became possible with the arrival of PDM systems. PDM systems do not only manage geometric data, they also offer product information such as parts lists. Parts lists are normally managed by larger database systems. By complete integration (Figure 6) of the PDM and CAD systems, geometric data can be converted into VRML data without having to interact with the CAD system. Selected objects are actually converted into VRML and meta-data in one integrated operation, called transfer process (Figure 6). This process is similar to the VRLM-converter and the meta-data file generator, put together.

There is one major difference between the CAD- and the PDM-connection. As soon as a PDM user selects an object, a pointer is set on the corresponding parts list. This pointer is stored in the object's meta-data. Supported by such pointers, it is possible, at any time, to load original parts lists and geometric data from the PDM system and to display them with the CAD system. 
Connecting PDM with 3D-CAD and BUILD-IT systems, opens up new possibilities, far beyond managing parts list and geometric data. The main advantage of this combination is the concept of parts list integration (Figure 6). Parts list integration means bi-directional communication between the PDM system and BUILD-IT. Henceforth, it is possible to harvest the full advantages of a BUILD-IT planning session.

BUILD-IT users can assemble objects without having to care about causing any harm to original parts lists or geometric data. As soon as a planning process is accomplished, BUILD-IT generates a co-ordinates list. The list is the final result of the planning session and describes all the assembled objects. Supported by the parts list integration, communicated via the PDM system, the CAD system can now access, integrate and display the result of the planning session. Object modification that took place during the BUILD-IT planning session has no effect beyond that session.

\section{USER EXPERIENCES}

The BUILD-IT system was tried out with managers and engineers from companies producing assembly lines and plants. These tests showed that the system is easy to handle, intuitive and enjoyable to use. Most people were able to assemble virtual plants after only a few minutes of introduction. Some typical user comments were: 1) "The concept phase is especially important in plant design since the customer must be involved in a direct manner. Often, partners using different languages sit at the same table. This novel interaction technique will be a means for completing this phase efficiently." 2) "This is a general improvement of the interface to the customer, in the offering phase as well as during the project, especially in simultaneous engineering projects." 3) "The use of this novel interaction technique will lead to simplification, acceleration and reduction of the iterative steps in the start-up and concept phase of a plant construction project".

For the development of specific scenarios for each type of design task, we carried out interviews. Our subjects were expert designers, and the aim was to elicit their planning strategies, in order to get hold of relevant interactive parameters for a planning session.

\section{DISCUSSION AND FUTURE PERSPECTIVES}

Apart from enriching human-computer interaction in a direct and simple way, BUILD-IT has three further advantages over other Virtual Reality (VR) systems. 
First, BUILD-IT supports group interaction while other systems, such as immersive VR, are single-user. Second, with its mixture of virtual and real tools, BUILD-IT allows for mixed (real and virtual) interaction, whereas other systems either use a mouse (pointer) or purely virtual tools. Third, BUILD-IT encourages teamwork among real persons interacting with real objects. All topics will be subjects for HCI research in general, and for the further development of BUILDIT into an industry standard product in particular.

Plans for further development of BUILD-IT has been divided into three stages:

- Task analysis and interaction design: This stage will explore various ways of interaction, considering the task to be performed. It also includes preliminary experiments for cost/benefit studies of various forms of implementation, e.g. computer performance vs. group symbioses and user interaction. By the end of this stage, after approximately one year, various configurations of a functional BUILD-IT system, consisting of hardware and software, should be available.

- User evaluation: The second stage will consist of comparing the various configurations through usability studies. The aim is to investigate the relative advantage of different configurations relative to the task performed and to investigate the advantage of BUILD-IT vs. conventional desk-top systems, also relative to the task performed.

- Prototyping: Throughout the two first stages, the realisation can be at the level of wood and wire solutions. The third stage will consist of developing these preliminary systems into a commercial product.

\section{REFERENCES}

AESOP GmbH (1997) SIMPLE++ for modelling, simulation and visualisation.

Ackermann, P. (1996) Developing Object-Oriented Multimedia Software Based on the MET++ Application Framework. Heidelberg: dpunkt Verlag für digitale Technologie.

Fjeld, M., Bichsel, M. and Rauterberg, M. (1998) BUILD-IT: An Intuitive Design Tool Based on Direct Object Manipulation. In: Wachsmut, I. and Frölich, M. (eds.) Gesture and Sign Language in Human-Computer Interaction, Lecture Notes in Artificial Intelligence 1371, Springer, Heidelberg, pp. 297-308.

Hacker, W. (1994) Action regulation theory and occupational psychology. Review of German empirical research since 1987. The German Journal of Psychology 18(2) pp. 91-120.

Ishii, H. and Ullmer, B. (1997) Tangible Bits: Towards Seamless Interfaces between People, Bits and Atoms. In the Proceedings of CHI'97.

Kirsh, D. and Maglio, P. (1994) On Distinguishing Epistemic from Pragmatic Action, Cognitive Science, 18, 513-549.

Rauterberg, M., Mauch, T. \& Stebler, R. (1996) What is a promising candidate for the next generation of interface technology. In Proceedings of 5 th International Conference INTERFACE to Real \& Virtual Worlds' (pp. 95-103). Montpellier: EC2 \& Cie. 
Underkoffler, J. and Ishii, H. (1998) Illuminating Light: An Optical Design Tool with Physical Interface. In Proceedings of CHI'98.

\section{BIOGRAPHIES}

Morten Fjeld

1990

1990-1997

$1997-$
: MSc in Applied Mathematics, Norwegian Institute of Technology

: Design and realisation of real-time, industrial simulators, measuring systems and training equipment

$: \mathrm{PhD}$ student and research assistant in Cognitive Sciences and Human-Computer Interaction at ETH Zurich

Kristina Lauche

1995

$1995-$

: MSc in Psychology, Free University Berlin

$1997-$

: PhD student in Psychology

: Research assistant in Psychology at ETH Zurich

Stefan Dierssen 1997

: MSc in Mechanical En gineering, Technical University Clausthal 1997 -

: PhD student and research assistant in Product Data

Management at ETH Zurich

Martin Bichsel

1987

: MSc in Physics, ETH Zurich

1991

: PhD in Physics, ETH Zurich

1991-1992

: Postdoctoral fellowship at Media Laboratory, MIT Boston

1992-1996

: Senior lecturer in Computer Science at University of Zurich

1997 -

: Senior lecturer in Computer Vision and Graphics at ETH Zurich

Matthias Rauterberg

1981

1985

1995

1995-1998
: MSc in Psychology, University of Hamburg

: MSc in Computer Science, University of Hamburg

: PhD in Computer Science, ETH Zurich

: Senior lecturer in Computer Sci ence and Industrial

Engineering at ETH Zurich

1998-
: Professor in Human Communication Technology at the Center for Research on User-System Interaction (IPO), TU Eindhoven 